\title{
The Labile Iron Pool in Monocytes Reflects the Activity of the Atherosclerotic Process in Men With Chronic Cardiovascular Disease
}

\author{
P. RIŠKO ${ }^{1}$, J. PLÁTENÍK ${ }^{2}$, R. BUCHAL ${ }^{2}$, J. POTOČKOVÁ $^{1}$, P. J. KRAML ${ }^{1}$
}

${ }^{1}$ Second Department of Internal Medicine, Faculty Hospital Královské Vinohrady and Third Faculty of Medicine, Charles University, Prague, Czech Republic, ${ }^{2}$ Institute of Medical Biochemistry and Laboratory Diagnostics, First Faculty of Medicine, Charles University, Prague, Czech Republic

Received April 30, 2016

Accepted June 22, 2016

On-line October 26, 2016

\section{Summary}

The study investigates the relationship between the labile iron pool (LIP) in circulating monocytes and markers of iron metabolism, inflammation, oxidative stress, endothelial dysfunction and arterial elasticity in patients with chronic cardiovascular disease and in healthy volunteers. The patients with a history of CVEs had significantly higher LIP values than did the control group $(1.94 \pm 0.46 \mu \mathrm{M}$ vs. $1.62 \pm 0.49 \mu \mathrm{M}, \mathrm{p}=0.02)$. Except for the leukocyte number (WBCs), the groups did not differ in other inflammatory markers (CRPus, CD 163, MPO, MMP-1). Similarly, there were no differences in the markers of endothelial dysfunction (ICAM, VCAM, E-selectin, VWF). The CVE group had higher pulse pressures, levels of markers of impaired arterial elasticity (AI, Young's modulus, pulsatility, stiffness index), IMT values and ABI values. The LIP concentration was significantly correlated with the transferrin receptor/ferritin ratio, hepcidin levels, VFT content and the $A B I$ and ET values. Patients with a history of CVE have significantly higher concentrations of iron in their intracellular LP in circulating monocytes than do healthy controls. The independent and significant correlation of LP with markers of the progression of atherosclerosis and arterial stiffness suggests LP as a possible novel marker of atherosclerotic activity.

\section{Key words}

Iron overload • LIP • Monocytes • Inflammation • Arterial stiffness

\section{Corresponding author}

P. Riško, Second Department of Internal Medicine, Faculty Hospital Královské Vinohrady, Šrobárova 1150/50, 10034 Praha 10, Czech Republic. E-mail: riskopeter1985@gmail.com or riskopeter@seznam.cz

\section{Introduction}

Iron as a cardiovascular risk factor has been discussed since the early 1980s. Sullivan (1981) was apparently the first who proposed that iron overload contributes to atherosclerosis and promotes cardiovascular disease. The presence of redox-active iron as well as elevated expression of $\mathrm{H}$ - and L-ferritin in human atherosclerotic plaques further supports the iron hypothesis (Smith et al. 1995, Pang et al. 1996). The results of several human epidemiological studies strongly suggest an association of the body's iron stores with cardiovascular mortality (Lauffer et al. 1990) and higher risks of myocardial infarction (Salonen et al. 1992, Tuomainen et al. 1998) or peripheral vascular disease (Menke et al. 2009). Kiechl et al. suggested serum ferritin levels as an indicator of carotid atherosclerotic progression (Kiechl et al. 1997). In a previous study, we described a significant correlation of stored iron levels with asymptomatic carotid atherosclerosis in healthy men of primary prevention (Syrovatka et al. 2011).

Iron is an essential element in the human body that plays an important role in oxygen metabolism, electron transfer, and acts as a cofactor for many enzyme systems. Under physiological conditions, the body keeps a stable pool of iron. Most of the surplus iron is stored in hepatocytes and in the mononuclear phagocyte system (MPS). The MPS (monocytes, macrophages and their precursors) plays a major role in the recycling of the body's iron pool (Kong et al. 2008).

Inflammation is a characteristic feature of atherosclerosis. Macrophages are a major type of 
inflammatory cell and play a crucial role in the development and progression of the atheromatous plaque. M1 macrophages are present in human atherosclerotic lesions (Khallou-Laschet et al. 2010), where they produce proinflammatory cytokines (TNF $\alpha$, IL-1 $\beta$, IL-6) contributing to local inflammation and the progression and eventual destabilization of the plaque. During plaque hemorrhage, the pro-oxidant environment of atherosclerotic tissue promotes erythrocyte lysis and the accumulation of free hemoglobin, which, unless eliminated, may cause tissue damage via oxidative stress (Habib and Finn 2014).

Iron within the plaque undoubtedly modifies the immunoreactivity and metabolism of macrophages/monocytes. However, the molecular mechanisms underlying the iron homeostasis, metabolism and immune activity of the MPS are poorly understood. High iron stores are associated with activation of both humoral and cellular immunity (De Palma et al. 2003). Iron and iron-binding proteins modulate the functions of $\mathrm{B}$ and $\mathrm{T}$ lymphocytes, macrophages and natural killer cells (NK cells) (Feng et al. 1994, Kemp 1993, Bovlus 2003). Iron seems to have a direct impact on macrophage activation and on their transformation to foam cells (Yuan et al. 2004).

Accordingly, it seems evident that the iron content of macrophages has the potential to significantly affect the atherosclerotic process. Most of intracellular iron is located in ferritin, but some can exist in a loosely bound condition as the cytosolic labile iron pool (LIP) (Greenberg and Wintrobe et al. 1946, Jacobs 1997), which is thought to represent the redox-active iron capable of catalyzing the formation of oxygen free-radicals. The LIP is a highly exchangeable pool of iron ions that sits on the crossroads of the metabolic pathways of iron-containing compounds (Kruszewski 2003) and is associated with a diverse population of ligands, such as phosphates, carboxylates, polypeptides, and the polar groups of membrane phospholipids (Kakhlon et al. 2002).

Our long-standing scientific interest is focused on the role of the body's iron pool in the pathogenesis of cardiovascular diseases. In this study, we investigate a possible relationship between the LIP in circulating monocytes and other parameters of iron metabolism, inflammation, endothelial dysfunction and arterial elasticity in patients with a history of cardiovascular disease and in healthy volunteers.

\section{Materials and Methods}

\section{Patients and controls}

A total of 51 men volunteers were recruited into the study. The cohort of volunteers with cardiovascular events (patients after AMI or stroke, at least 3 months after the last acute coronary syndrome, with peripheral arterial disease) were recruited from the patients registered in the Department of Preventive Cardiology. The control group of healthy individuals were recruited from the volunteers with no history of CVE to obtain age matched groups. All respondents provided written informed consent, and the study was approved by the ethics committee of the 3rd Medical Faculty, Charles University, Prague. Exclusion criteria were an age over 65 years; any acute or chronic inflammatory disease or hemorrhagic diathesis; current use of immunosuppressants, non-steroidal anti-inflammatory drugs or chelates; and any surgery or transfusions of blood derivatives within last 3 months. The medical history and detailed information about previous cardiovascular diseases were obtained. All participants filled out Baecke's habitual questionnaire focused on dietary habits and physical activity (Baecke et al. 1982, Florindo et al. 2006). Anthropometric measurements included body height, weight, and waist and hip circumferences. The body mass index (BMI) and waistto-hip ratio (WHR) were calculated. Resting blood pressure (systolic/diastolic), ankle-brachial index (ABI), pulse pressure (PP), and mean arterial pressure (MAP) were measured/calculated in all respondents. We measured the cardio-ankle vascular index (CAVI), augmentation index (AI), pre-ejection time (PEP), and ejection time (ET) using a VaSera VS-1500N system (Fukuda Denshi).

\section{Ultrasound measurements}

High-resolution B-mode ECG-gated ultrasonography of both common carotid arteries was performed in all subjects while in the supine position. The extracranial carotid arteries were examined bilaterally using a Philips iU22 ultrasound system equipped with a $7.5 \mathrm{MHz}$ linear array transducer, and analyzed using QLAB Quantification software (Philips). The intima-media thickness (IMT) was measured in diastole on the far walls of the common carotid arteries at a distance of $10 \mathrm{~mm}$ from the carotid bulb after $10 \mathrm{~min}$ of rest (Stein et al. 2008). Young's modulus (YEM), stiffness parameter $\beta$ (SI), pulsatility index (PI) and 
resistance index (RI) were calculated for an estimation of arterial elasticity (Cavallini et al. 1996, Mitsumura et al. 2014).

Transverse scanning was performed to measure the maximum visceral fat thickness (VFT) using a 3.5-MHz ultrasound probe. Measurements were obtained $1 \mathrm{~cm}$ above the umbilicus. VFT was defined as the distance between the anterior wall of the aorta and the internal face of the rectoabdominal muscle perpendicular to the aorta. Images were obtained immediately after a calm and complete expiration to avoid the influence of the respiratory status or abdominal wall tension (de Lucia Rolfe et al. 2010). Measurements were performed by the same person three times and averaged.

\section{Biochemical parameters}

Blood samples were collected from the subjects early in the morning after at least $10 \mathrm{~h}$ of fasting. The samples were sent to the Department of Laboratory Diagnostics FNKV for the analysis of routine parameters (lipid profile (total cholesterol (TC), triglycerides (TGs), high-density lipoprotein (HDL-C), apolipoprotein A1 (ApoA1), apolipoprotein B (ApoB), lipoprotein a (Lp(a)), blood count and differential, glucose (GLC), C-peptide, insulin (INS), urea, creatinine, bilirubin, uric acid, minerals ( $\mathrm{Na}, \mathrm{K}, \mathrm{Cl}, \mathrm{Ca}, \mathrm{P}, \mathrm{Mg}$ ), liver enzymes (alanine aminotransferase (ALT), aspartate aminotransferase (AST), gamma-glutamyl transferase (GGT), alkaline phosphatase (ALP)), and iron-associated parameters (Fe, binding capacity of Fe (TBIC), ceruloplasmin). For the isolation of circulating monocytes 3 samples (6 ml each) of blood were taken with $\mathrm{K}_{3}$ EDTA used as an anticoagulant. All efforts were made to minimize hemolysis. Within one hour, the blood samples were transported on ice to the laboratory where the isolation of monocytes for LIP measurement started immediately. Samples for the remaining analyses were immediately isolated by centrifugation and stored at $-80{ }^{\circ} \mathrm{C}$ until further analysis. Ferritin (L- and H-chains), transferrin and circulating transferrin receptors were measured using a commercial ELISA kit from USCN (Cloud Clone Corp./Wuhan USCN Business Co., Ltd., Houston, TX 77084, USA); hepcidin, an ELISA kit from DRG (DRG Diagnostics GmbH, Marburg, Germany); oxidized lowdensity lipoprotein (OxLDL) and myeloperoxidase (MPO), a kit from Mercodia (Mercodia AB, Uppsala, Sweden); anti-oxLDL, a kit from Biomedica (Biomedica Medizinprodukte, GmbH, Vienna, Austria); matrix metalloproteinase-1 (MMP-1), a kit from RayBiotech
(RayBiotech Inc., Norcross, GA, USA); intercellular adhesion molecule 1 (ICAM-1), a Platinum kit from eBioscience (Bender MedSystems GmbH, Vienna, Austria); vascular cell adhesion molecule 1 (VCAM-1) and CD163, a kit from R\&D Systems (R\&D Systems, Inc., Minneapolis, MN, USA); E-selectin, von Willebrand factor (vWF) and transforming growth factor beta 1 (TGF-1beta), a kit from Abcam (Abcam, Cambridge, UK); ultrasensitive C-reactive protein (CRPus), a kit a kit from Roche using a COBAS 8000 analyzer (Department of Laboratory Diagnostics FNKV); $\mathrm{Cu}$, using F-AAS (flame atomic absorption spectrometry) (SPADIA LAB, a.s.). Low-density lipoprotein (LDL) was calculated and also measured using a kit from Roche/Hitachi (COBAS c, LDL-cholesterol plus, 2nd generation, COBAS 8000 analyzer) (Department of Laboratory Diagnostics FNKV).

The insulin resistance index (homeostasis model assessment of insulin resistance, HOMA-IR) was calculated using the HOMA2 glucose homeostasis model (Levy et al. 1998). The atherogenic index of the plasma was calculated as the log[triglycerides/high-density lipoprotein (HDL) cholesterol] (Dobiasova and Frohlich 2001).

Isolation of circulating monocytes, measurement of cytosolic LIP and cellular ferritin

\section{Reagents and antibodies}

OptiPrep $^{\mathrm{TM}}(60 \%$ (w/v) iodixanol in water) was obtained from Axis-Shield Density Gradient Media (Alere Technologies AS, Oslo, Norway). The PCV Packed Cell Volume Tubes and "easy read" measuring device were purchased from TPP AG (Trasadingen, Switzerland). Calcein and its acetoxymethyl (AM) ester were manufactured by Molecular Probes (Thermo Fisher Scientific, Waltham, MA). Salicylaldehyde isonicotinoyl hydrazone $(\mathrm{SIH})$ was a generous gift from Prof. Prem Ponka, McGill University, Montreal, Canada. Mouse monoclonal antibody to CD14, biotin conjugated (1B-293-C100) and mouse monoclonal IgG1 isotype control, biotin conjugated (1B-632-C100) were obtained from EXBIO Praha (Vestec u Prahy, Czech Republic). The Vectastain Elite ABC kit and 3,3'-diaminobenzidine (DAB) substrate kit were provided by Vector Laboratories, Inc. (Burlingame, CA). The PVDF blotting membrane used was the Immobilon-P from Millipore (Billerica, MA). The $\beta$-actin rabbit monoclonal antibody, HRP conjugate (\#5125), FTH1 (H-ferritin) rabbit 
monoclonal antibody (\#4393), and anti-rabbit IgG HRPlinked antibody (\#7074) were from Cell Signaling Technology, Inc. (Danvers, MA). The WesternBrightTM Quantum chemiluminescent HRP substrate was obtained from Advansta Inc. (Menlo Park, CA). Sodium dodecyl sulfate (SDS) was purchased from SERVA Electrophoresis GmbH (Heidelberg, Germany). Cell culture medium (RPMI 1640, Dutch modification, with $1 \mathrm{~g} / \mathrm{l} \mathrm{NaHCO}_{3}, 20 \mathrm{mM}$ HEPES, without glutamine), bovine serum albumin (BSA), HEPES, dimethyl sulfoxide (DMSO) and CC/MountTM aqueous mounting medium and other chemicals used were from SigmaAldrich (St. Louis, MO).

\section{Isolation of monocytes from peripheral blood}

We adopted the flotation method developed by Graziani-Bowering et al. (1997) with modifications for use with whole blood (Isolation of a monocyte 2013). Typically, blood samples from two subjects were processed in parallel. Samples and all solutions were precooled to $4{ }^{\circ} \mathrm{C}$ and kept on ice throughout the procedure.

A $1.070 \mathrm{~g} / \mathrm{ml}$ density barrier-solution was prepared by mixing $10 \mathrm{ml}$ of OptiPrep ${ }^{\mathrm{TM}}$ with $40 \mathrm{ml}$ of RPMI 1640 and $0.5 \%(\mathrm{w} / \mathrm{v})$ BSA (RPMI-BSA) and $54 \%(\mathrm{w} / \mathrm{v})$ iodixanol was made by combining $7.2 \mathrm{ml}$ of OptiPrepTM with $0.8 \mathrm{ml}$ of $8.5 \%$ (w/v) NaCl. Venous blood (16 ml) was gently mixed with $3.2 \mathrm{ml}$ of the $54 \%$ iodixanol and dispensed in $4 \mathrm{ml}$ aliquots into 15-ml polypropylene tubes. $6 \mathrm{ml}$ of the density barrier solution was carefully layered over the blood in each tube, followed by $0.5 \mathrm{ml}$ of RPMI-BSA placed on top. The gradients were centrifuged at $700 \mathrm{~g}$ in a swinging bucket rotor for $30 \mathrm{~min}$ at $4{ }^{\circ} \mathrm{C}$ with slow deceleration. The monocytes were collected from the upper interface, diluted with 4 volumes of RPMI-BSA and centrifuged at $300 \mathrm{~g}$ for $10 \mathrm{~min}$ at $4{ }^{\circ} \mathrm{C}$. The resulting cell pellet was resuspended in $2 \mathrm{ml}$ of RPMI-BSA and kept on ice.

Nucleated elements were counted in a hemocytometer chamber with Türk's solution. The cell suspension was then used measure the LIP as described below. Smears were prepared for CD14 staining and the volume of cells was estimated using the PCV tubes. The remaining cells were denatured for Western immunoblotting by mixing 4 volumes of the cell suspension with 1 volume of $50 \mathrm{~g} / \mathrm{l}$ SDS and $0.5 \mathrm{M}$ dithiothreitol and boiling for $10 \mathrm{~min}$.

The isolation procedure described above reproducibly yielded a suspension of fully viable mononuclear cells, free from granulocytes and platelets. On average, $2.2 \times 10^{6}$ cells were obtained from $10 \mathrm{ml}$ of blood. According to CD14 staining, the mean purity was $56 \%$; the other cells appeared to be lymphocytes. The average recovery was $22 \%$ relative to the original number of monocytes in the blood samples. Erythrocytes were never found in the preparations. However, a quantitatively minor $(<10 \%)$ contamination with fragments of red blood cells was present in about half of the cases, visible as a reddish upper rim on the cells pelleted in the PCV columns.

\section{Measurement of the LIP in isolated monocytes}

The concentration of the LIP in isolated monocytes was measured according to Epsztejn et al. (1997). Briefly, $10^{6}$ isolated cells in $2 \mathrm{ml}$ RPMI-BSA were loaded with $0.25 \mu \mathrm{M}$ calcein AM ester for 5 min at $37^{\circ} \mathrm{C}$, resuspended in RPMI-BSA, and transferred to $4 \mathrm{ml}$ of HEPES-buffered saline (20 mM HEPES/ $\mathrm{NaOH}$ at pH 7.3 and $150 \mathrm{mM} \mathrm{NaCl}$ ). Three milliliters of the cell suspension in a spectrofluorimetric quartz cuvette with magnetic stirrer were placed in the thermostated cuvette holder of a Fluoromax 3 spectrophotometer (HORIBA Jobin-Yvon S.A.S., France). Fluorescence of the cell suspension was monitored at an excitation wavelength of $488 \mathrm{~nm}$ and emission wavelength of $510 \mathrm{~nm}$ with constant stirring at $25^{\circ} \mathrm{C}$. The background signal was recorded for $400 \mathrm{~s}$ (F1), followed by measurement with $100 \mu \mathrm{M}$ of SIH for $300 \mathrm{~s}$ (F2), and with a suitable amount of a standard solution of calcein for another $200 \mathrm{~s}$ (F3). The standard was prepared by dissolving calcein (free acid) in $50 \mathrm{mM} \mathrm{K}_{2} \mathrm{HPO}_{4}$ to approximately $1.6 \mu \mathrm{M}$. The exact concentration was determined spectrophotometrically $\left(\varepsilon=77,000 \mathrm{~cm}^{-1} \mathrm{M}^{-1}\right.$ at $494 \mathrm{~nm}$, based on the manufacturer's data). The standard was aliquoted and stored at $-20^{\circ} \mathrm{C}$ and carefully protected from light; new aliquots was used on each measurement day.

The mean values of F1, F2 and F3 were obtained from the last $150 \mathrm{~s}$ of each trace and used to calculate the LIP ( $\mu$ M) according to Epsztejn et al. (1997), including the dissociation constant of the iron-calcein complex $\left(K_{d}=0.22 \mu M\right)$. The cell volume needed for the calculation was measured using the PCV columns.

\section{Immunocytochemical staining for CD14}

The smears of isolated cells on glass slides were fixed in freshly prepared ice-cold $3.7 \%$ (v/v) formaldehyde in phosphate-buffered saline (PBS) for $10 \mathrm{~min}$. The slides were blocked in $1 \mathrm{~g} / \mathrm{l} \mathrm{BSA}$ in PBS 
(PBS-BSA) for $30 \mathrm{~min}$ and incubated with either an antibody against CD14 or an IgG1 isotype control antibody (negative control) at a 1:500 dilution in PBS-BSA for $30 \mathrm{~min}$. The slides were then developed with the $A B C$ reagent and the $\mathrm{DAB}$ substrate as directed by the manufacturer and, finally, mounted with CC/MountTM mounting medium.

The stained smears were examined with an inverted microscope equipped with a digital camera. Three random digital images were captured at 200× in both phase contrast and bright field modes and used to count the cells to determine the mean proportion of CD14+ cells (purity) for each preparation.

Western immunoblotting for ferritin in isolated monocytes

The samples of SDS-denatured monocytes were applied to $4.5 \%-12.5 \%$ SDS-polyacrylamide gels, together with a standard prepared by mixing several samples with approximately average cell concentrations. The proteins were resolved via electrophoresis (15 mA/gel) and transferred onto a PVDF blotting membrane. The blots were blocked for one hour with $5 \%$ (w/v) skim milk in Tris-buffered saline with $0.05 \%$ (w/v) Tween 20 (TBST) and then incubated with antibodies against human $\mathrm{H}$-ferritin at a 1:1,000 dilution in $5 \%$ (w/v) skim milk in TBST overnight at $4{ }^{\circ} \mathrm{C}$ on a tilting platform, followed by secondary an incubation with HRP-conjugated anti-rabbit antibody at a dilution of 1:1,000 in $1 \%(\mathrm{w} / \mathrm{v})$ skim milk in TBST for one hour at room temperature. The blots were then washed with TBST and developed with the WesternBright ${ }^{\mathrm{TM}}$ Quantum chemiluminescent HRP substrate, as directed by the manufacturer. The chemiluminescence was read using a Fusion FX7 instrument (Vilber Lourmat, Marne-la-Vallée, France) and evaluated with Vilber Lourmat Bio-1D software version 15.03. The volumes of the bands were obtained from an optimally exposed picture and normalized to the signal of the standard present in each gel. The blots were then subjected to another detection with the HRP-linked antibody against human $\beta$-actin at a dilution of 1:1,000 in $5 \%(\mathrm{w} / \mathrm{v})$ skim milk in TBST. Initially, $6 \mu \mathrm{l}$ (30 $\mu \mathrm{g}$ of BSA) of each sample was applied; the results were used to calculate the equivalent sample amounts and all samples were reassayed. Both the $\mathrm{H}$-ferritin and actin signals were normalized to the standard present in each gel, and for each sample, values are reported as the H-ferritin/actin ratio.

\section{Statistical analysis}

Statistical analyses were performed by a professional statistician using Statistica 12.0 (StatSoft, USA). Comparison of the groups was performed using Student's $t$-test for two independent samples. For variables with a nonhomogeneous distribution, the MannWhitney U test was used. Pearson's or Spearman's correlation analyses were used to assess any potential relationships between the LIP and other variables. Statistical significance was defined as $\mathrm{P}<0.05$.

\section{Results}

The basic characteristics of the volunteers are shown in Table 1 (A, B, C, D). The groups did not differ in basic characteristics, such as age, weight, BMI or waist circumference, but CVE patients had thicker visceral fat tissue. The CVE patient group also had a significantly higher pulse pressure, although no differences in blood pressure were observed (Table 1A). For the basic biochemical parameters, we found significantly lower concentrations of TC, LDL, and apo-B in the CVE group than in the control group. CVE patients had higher concentrations of TGs and lower concentrations of HDL than the healthy volunteers. Moreover, there were also significantly higher levels of plasma glucose, C-peptide and insulin and a higher calculated insulin resistance index (HOMA-IR) in the CVE patient group (Table 1B). The patients with a history of CVE demonstrated significantly higher values for the LIP even though there was no difference in other markers of iron metabolism. Additionally, CVE individuals had higher concentrations of copper and ceruloplasmin than the healthy individuals (Table 1C).

Except for the number of leukocytes (WBCs), there was no difference in the other measured inflammatory parameters (CRPus, CD 163, MPO, MMP-1) between the groups. Similarly, the cohorts did not differ in the common markers of endothelial dysfunction (ICAM, VCAM, E-selectin, vWF) or in the parameters related to LDL oxidation. However, a significantly lower level of anti-oxLDL antibodies in CVE patients was found (Table 2).

As expected, the CVE patient group showed higher levels of markers of impaired arterial elasticity (AI, Young's modulus, PI, SI) markers of atherosclerotic progression (IMT, ABI). The CVE patients also had thicker visceral fat measured by ultrasound (Table 3).

Because of the significant differences in the 
concentrations of the LIP, we calculated the correlation of the LIP with other measured parameters. Of the factors related to iron metabolism, the LIP correlated significantly with the TfR/F ratio and with hepcidin. Of the measured inflammatory parameters, the LIP correlated only with the number of WBCs. Nevertheless, the LIP showed a significant correlation with ABI, the marker of atherosclerotic progression, and ET, the parameter of arterial stiffness. Interestingly, the LIP was positively correlated with VFT (Table 4).

Multivariate regression analysis confirmed that each correlation of the LIP was independent of the other correlated parameters except for LIP's correlation with the VFT (Table 5).

Further analysis showed that the OR for acute CVE was 6 fold higher when LIP tertiles were compared $(\mathrm{OR}=5.96, \mathrm{P}=0.0156)$ (Table 6).

Table 1. The basic characteristics of the volunteers (A, B). Basic biochemical parameters (C). Parameters of iron and copper metabolism (D).

A)

\begin{tabular}{|c|c|c|c|c|c|}
\hline & \multicolumn{2}{|c|}{ Healthy group } & \multicolumn{2}{|c|}{ CVE group } & \multirow{2}{*}{$\mathbf{P}$} \\
\hline & $\mathrm{n}$ & $\mathrm{x} \pm \mathrm{SD}$ & $\mathrm{n}$ & $\mathrm{x} \pm \mathrm{SD}$ & \\
\hline Age (years) & 28 & $54.56 \pm 6.62$ & 23 & $57.27 \pm 5.44$ & 0.1223 \\
\hline Weight (kg) & 28 & $88.30 \pm 18.00$ & 23 & $91.83 \pm 15.38$ & 0.4613 \\
\hline$B M I\left(\mathrm{~kg} / \mathrm{m}^{2}\right)$ & 28 & $27.93 \pm 4.67$ & 23 & $29.95 \pm 4.81$ & 0.1357 \\
\hline Waist (cm) & 27 & $96.50 \pm 13.75$ & 23 & $102.15 \pm 11.52$ & 0.1255 \\
\hline Hips (cm) & 27 & $101.44 \pm 11.43$ & 23 & $101.70 \pm 7.79$ & 0.9293 \\
\hline Visceral fat thick. $(\mathrm{cm})$ & 28 & $6.13 \pm 2.22$ & 23 & $8.52 \pm 2.86$ & 0.0015 \\
\hline FAT subcut. (cm) & 28 & $2.40 \pm 0.84$ & 23 & $2.18 \pm 1.00$ & 0.3994 \\
\hline Systolic BP (mmHg) & 28 & $132.96 \pm 19.00$ & 23 & $141.62 \pm 11.75$ & 0.0627 \\
\hline Diastolic BP (mmHg) & 28 & $87.14 \pm 14.67$ & 23 & $88.47 \pm 8.62$ & 0.7033 \\
\hline Pulse pressure & 28 & $45.8 \pm 7.10$ & 23 & $53.15 \pm 8.53$ & 0.0016 \\
\hline
\end{tabular}

B)

\begin{tabular}{|c|c|c|c|c|}
\hline \multirow{2}{*}{ Diseases, risk factors } & \multicolumn{2}{|c|}{ Healthy group (28 men) } & \multicolumn{2}{|c|}{ CVE group (23 men) } \\
\hline & Num. of cases & $\%$ & Num. of cases & $\%$ \\
\hline History of stroke & 0 & 0.0 & 1 & 4.3 \\
\hline History of AIM & 0 & 0.0 & 14 & 60.9 \\
\hline Peripheral arterial disease & 0 & 0.0 & 10 & 43.5 \\
\hline Ischemic heart disease & 0 & 0.0 & 17 & 73.9 \\
\hline Effort angina pectoris & 0 & 0.0 & 2 & 8.7 \\
\hline Hypertension & 6 & 21.4 & 16 & 69.6 \\
\hline Diabetes mellitus 1. type & 0 & 0.0 & 0 & 0.0 \\
\hline Diabetes mellitus 2. type & 1 & 3.6 & 10 & 43.5 \\
\hline Hyperlipidemia & 4 & 14.3 & 18 & 78.3 \\
\hline Active smokers & 21 & 75.0 & 4 & 17.4 \\
\hline Former smokers & 2 & 7.1 & 9 & 39.1 \\
\hline Positive FH for CVE & 4 & 14.3 & 4 & 17.4 \\
\hline \multicolumn{5}{|l|}{ Medication } \\
\hline \multicolumn{5}{|l|}{ Antihypertensives } \\
\hline$B B$ & 2 & 7.1 & 16 & 69.6 \\
\hline
\end{tabular}




\begin{tabular}{lcccc}
\hline CCB & 2 & 7.1 & 6 & 26.1 \\
ACE/ARB & 3 & 10.7 & 17 & 73.9 \\
Diuretics & 0 & 0 & 7 & 30.4 \\
Others & 3 & 10.7 & 3 & 13.0 \\
Antiplatelet agents & 0 & 0 & 18 & 78.3 \\
Lipid lowering drugs & & & & \\
Statins & 3 & 10.7 & 23 & 100 \\
Fibrates & 0 & 0 & 5 & 21.7 \\
Ezetimib & 0 & 0 & 7 & 30.4 \\
Antidiabetics & & & & \\
PAD & 1 & 3.6 & 7 & 30.4 \\
Insulin & 0 & 0 & 1 & 4.3 \\
\hline
\end{tabular}

C)

\begin{tabular}{|c|c|c|c|c|c|}
\hline & \multicolumn{2}{|c|}{ Healthy group } & \multicolumn{2}{|c|}{ CVE group } & \multirow{2}{*}{$\mathbf{P}$} \\
\hline & $\mathrm{N}$ & $\mathrm{x} \pm \mathrm{SD}$ & $\mathrm{N}$ & $\mathrm{X} \pm \mathrm{SD}$ & \\
\hline Urea $(\mathrm{mmol} / \mathrm{l})$ & 28 & $5.25 \pm 1.15$ & 23 & $5.57 \pm 1.45$ & 0.3887 \\
\hline Creatinine $(\mu \mathrm{mol} / \mathrm{l})$ & 28 & $92.6 \pm 11.86$ & 23 & $88.5 \pm 13.19$ & 0.2493 \\
\hline Uric acid $(\mu \mathrm{mol} / \mathrm{l})$ & 28 & $332.6 \pm 72.33$ & 23 & $339.5 \pm 79.86$ & 0.7475 \\
\hline$A L T(\mu k a t / l)$ & 28 & $0.54 \pm 0.22$ & 23 & $0.88 \pm 1.69$ & 0.2937 \\
\hline AST $(\mu k a t / l)$ & 28 & $0.49 \pm 0.13$ & 23 & $0.63 \pm 0.96$ & 0.4653 \\
\hline GMT ( $\mu k a t / l)$ & 28 & $0.50 \pm 0.34$ & 23 & $0.87 \pm 0.54$ & 0.0045 \\
\hline$A L P(\mu k a t / l)$ & 28 & $1.09 \pm 0.28$ & 23 & $1.25 \pm 0.42$ & 0.1158 \\
\hline Bilirubin $(\mu k a t / l)$ & 28 & $12.2 \pm 7.27$ & 23 & $10.39 \pm 9.11$ & 0.4410 \\
\hline Total cholesterol $(\mathrm{mmol} / \mathrm{l})$ & 28 & $5.13 \pm 0.58$ & 23 & $4.18 \pm 0.86$ & 0.0001 \\
\hline$H D L(\mathrm{mmol} / \mathrm{l})$ & 28 & $1.38 \pm 0.36$ & 23 & $1.15 \pm 0.39$ & 0.0389 \\
\hline LDL (mmol/l) - estim. & 28 & $3.54 \pm 0.62$ & 23 & $2.39 \pm 0.71$ & 0.0001 \\
\hline LDL (mmol/l) - calcul. & 28 & $3.15 \pm 0.65$ & 23 & $2.17 \pm 0.69$ & 0.0001 \\
\hline Triglycerides (mmol/l) & 28 & $1.33 \pm 0.55$ & 23 & $1.88 \pm 1.00$ & 0.0176 \\
\hline Apo A1 $(g / l)$ & 28 & $1.56 \pm 0.24$ & 23 & $1.51 \pm 0.30$ & 0.5128 \\
\hline Apo B $(g / l)$ & 28 & $1.05 \pm 0.16$ & 23 & $0.91 \pm 0.20$ & 0.0094 \\
\hline $\operatorname{Lp}(a)(g / l)$ & 28 & $22.4 \pm 63.22$ & 23 & $55.7 \pm 84.64$ & 0.1144 \\
\hline Glucose (mmol/l) & 28 & $5.08 \pm 0.99$ & 23 & $7.31 \pm 2.85$ & 0.0003 \\
\hline C-peptide (pmol/l) & 28 & $564.6 \pm 232.77$ & 23 & $773.5 \pm 337.07$ & 0.0120 \\
\hline Insulin (mU/l) & 28 & $10.1 \pm 6.40$ & 23 & $17.8 \pm 8.47$ & 0.0006 \\
\hline WBCs $\left(x 10^{9} / l\right)$ & 28 & $5.80 \pm 1.75$ & 23 & $7.77 \pm 2.02$ & 0.0005 \\
\hline$R B C\left(\times 10^{12} / l\right)$ & 28 & $4.86 \pm 0.30$ & 23 & $4.91 \pm 0.36$ & 0.5800 \\
\hline$H G B(g / l)$ & 28 & $146.8 \pm 10.86$ & 23 & $152.8 \pm 12.95$ & 0.0779 \\
\hline $\operatorname{HCT}(l)$ & 28 & $0.43 \pm 0.02$ & 23 & $0.44 \pm 0.03$ & 0.1507 \\
\hline $\operatorname{PLT}\left(\times 10^{9} / l\right)$ & 28 & $220.2 \pm 46.45$ & 23 & $228.8 \pm 60.43$ & 0.5696 \\
\hline$N E(\%)$ & 28 & $56.1 \pm 9.17$ & 23 & $57.1 \pm 13.98$ & 0.7650 \\
\hline$L Y(\%)$ & 28 & $31.4 \pm 7.83$ & 23 & $27.0 \pm 9.41$ & 0.0771 \\
\hline MO (\%) & 28 & $7.75 \pm 2.18$ & 23 & $8.50 \pm 3.07$ & 0.3156 \\
\hline HOMA IR & 28 & $2.35 \pm 1.65$ & 23 & $5.96 \pm 3.87$ & 0.0001 \\
\hline
\end{tabular}


D)

\begin{tabular}{|c|c|c|c|c|c|}
\hline & \multicolumn{2}{|c|}{ Healthy group } & \multicolumn{2}{|c|}{ CVE group } & \multirow{2}{*}{$\mathbf{P}$} \\
\hline & $\mathrm{n}$ & $\mathrm{x} \pm \mathrm{SD}$ & $\mathrm{n}$ & $\mathrm{x} \pm \mathrm{SD}$ & \\
\hline $\mathrm{Fe}(\mu \mathrm{mol} / \mathrm{l})$ & 28 & $18.05 \pm 6.04$ & 23 & $18.27 \pm 5.12$ & 0.8971 \\
\hline TIBC $(\mu \mathrm{mol} / \mathrm{l})$ & 28 & $54.43 \pm 9.80$ & 23 & $58.22 \pm 7.92$ & 0.1412 \\
\hline Ceruloplasmin (g/l) & 28 & $0.21 \pm 0.02$ & 23 & $0.23 \pm 0.03$ & 0.0166 \\
\hline $\mathrm{Cu}(\mu \mathrm{mol} / \mathrm{l})$ & 26 & $13.99 \pm 1.94$ & 22 & $15.64 \pm 2.34$ & 0.0105 \\
\hline Ferritin $(\mathrm{ng} / \mathrm{ml})$ & 25 & $129.09 \pm 122.90$ & 22 & $102.30 \pm 75.88$ & 0.3812 \\
\hline H-Ferritin (ng/ml) & 25 & $26.75 \pm 22.03$ & 22 & $24.12 \pm 18.42$ & 0.6618 \\
\hline L-Ferritin $(\mathrm{ng} / \mathrm{ml})$ & 25 & $10.49 \pm 3.13$ & 22 & $9.12 \pm 2.37$ & 0.1021 \\
\hline Transferrin $(\mathrm{mg} / \mathrm{ml})$ & 25 & $7.78 \pm 2.65$ & 22 & $8.13 \pm 3.08$ & 0.6765 \\
\hline Transferrin rec.(ng/ml) & 25 & $30.27 \pm 9.25$ & 22 & $30.40 \pm 8.80$ & 0.9587 \\
\hline Transferrin rec/Ferritin & 25 & $0.54 \pm 1.03$ & 22 & $0.45 \pm 0.39$ & 0.6938 \\
\hline Hepcidin $(n g / m l)$ & 25 & $18.35 \pm 5.75$ & 22 & $17.71 \pm 6.18$ & 0.7169 \\
\hline$L I P(\mu M)$ & 28 & $1.62 \pm 0.49$ & 23 & $1.94 \pm 0.46$ & 0.0200 \\
\hline $\begin{array}{l}\text { Cellular ferritin } \\
\text { (H-FerritinActin) }\end{array}$ & 28 & $1.25 \pm 1.75$ & 23 & $1.41 \pm 1.44$ & 0.7257 \\
\hline Yield of monocytes (\%) & 28 & $0.24 \pm 0.13$ & 23 & $0.23 \pm 0.05$ & 0.5499 \\
\hline
\end{tabular}

Table 2. Parameters of inflammation, endothelial dysfunction, oxidative stress.

\begin{tabular}{lccccc}
\hline & \multicolumn{2}{c}{ Healthy group } & \multicolumn{2}{c}{ CVE group } \\
& $\mathrm{n}$ & $\mathrm{x} \pm \mathrm{SD}$ & $\mathrm{n}$ & $\mathrm{x} \pm \mathrm{SD}$ & P \\
\hline CRP us $(\mathrm{mg} / \mathrm{l})$ & 28 & $1.62 \pm 1.397$ & 23 & $2.54 \pm 3.222$ & 0.1755 \\
CD163 $(\mathrm{ng} / \mathrm{ml})$ & 25 & $592.6 \pm 158.39$ & 22 & $647.4 \pm 257.51$ & 0.3781 \\
MPO $(\mathrm{\mu g} / \mathrm{l})$ & 25 & $75.3 \pm 115.31$ & 22 & $56.5 \pm 14.13$ & 0.4531 \\
MMP-1 $(\mathrm{pg} / \mathrm{ml})$ & 25 & $621.7 \pm 722.6$ & 22 & $940.4 \pm 963.6$ & 0.2029 \\
ICAM-1 $(\mathrm{ng} / \mathrm{ml})$ & 25 & $239.1 \pm 77.74$ & 22 & $273.4 \pm 59.35$ & 0.0992 \\
VCAM $(\mathrm{ng} / \mathrm{ml})$ & 25 & $841.5 \pm 205.77$ & 22 & $815.1 \pm 289.38$ & 0.7170 \\
vWF $(\mathrm{mU} / \mathrm{ml})$ & 25 & $1050.8 \pm 464.31$ & 22 & $901.1 \pm 519.49$ & 0.3046 \\
E-selectin $(\mathrm{ng} / \mathrm{ml})$ & 25 & $9.17 \pm 4.15$ & 22 & $10.7 \pm 5.85$ & 0.3117 \\
oxLDL $(\mathrm{U} / \mathrm{l})$ & 25 & $27.4 \pm 8.50$ & 22 & $27.0 \pm 7.79$ & 0.8529 \\
Anti-oxLDL $(\mathrm{mU} / \mathrm{ml})$ & 25 & $497.3 \pm 489.59$ & 22 & $245.0 \pm 292.60$ & 0.0405 \\
\hline
\end{tabular}

Table 3. Parameters of arterial elasticity and atherosclerotic progression.

\begin{tabular}{|c|c|c|c|c|c|}
\hline & \multicolumn{2}{|c|}{ Healthy group } & \multicolumn{2}{|c|}{ CVE group } & \multirow{2}{*}{$\mathbf{P}$} \\
\hline & $\mathrm{n}$ & $\mathrm{x} \pm \mathrm{SD}$ & $\mathrm{n}$ & $\mathrm{x} \pm \mathrm{SD}$ & \\
\hline$P I$ & 28 & $1.67 \pm 0.322$ & 23 & $2.024 \pm 0.591$ & 0.0098 \\
\hline$R I$ & 28 & $0.712 \pm 0.052$ & 23 & $0.743 \pm 0.069$ & 0.0800 \\
\hline$S I$ & 28 & $7.00 \pm 2.900$ & 23 & $10.29 \pm 3.768$ & 0.0010 \\
\hline$Y E M$ & 28 & $1399.3 \pm 658.4$ & 23 & $1875.2 \pm 791.4$ & 0.0231 \\
\hline CAVI & 28 & $8.00 \pm 1.018$ & 23 & $8.47 \pm 1.219$ & 0.1418 \\
\hline$A I$ & 28 & $1.04 \pm 0.188$ & 23 & $1.26 \pm 0.309$ & 0.0028 \\
\hline$E T$ & 28 & $301.9 \pm 21.00$ & 23 & $294.5 \pm 29.58$ & 0.3000 \\
\hline$P E P$ & 28 & $97.1 \pm 23.31$ & 23 & $105.9 \pm 22.52$ & 0.1777 \\
\hline
\end{tabular}




\begin{tabular}{lccccc}
\hline PEP/ET & 28 & $0.324 \pm 0.070$ & 23 & $0.364 \pm 0.100$ & 0.0945 \\
$A B I$ & 28 & $1.17 \pm 0.067$ & 23 & $0.951 \pm 0.155$ & 0.0001 \\
$I M T(\mathrm{~mm})$ & 28 & $0.560 \pm 0.097$ & 23 & $0.648 \pm 0.148$ & 0.0144 \\
\hline
\end{tabular}

Table 4. Univariate regression analysis of LIP's correlation with the measured parameters.

\begin{tabular}{|c|c|c|c|}
\hline Variable & $\mathbf{R}$ & Variable & $\mathbf{R}$ \\
\hline Age & -0.1220 & H-Ferritin & -0.1654 \\
\hline Weight & 0.0895 & L-Ferritin & -0.0664 \\
\hline$B M I$ & 0.1750 & H/L-Ferritin Ratio & -0.2075 \\
\hline Waist & 0.1892 & Ferritin & 0.2444 \\
\hline VFT & $0.3288 *$ & Transf. rec./Ferritin Ratio & $-0.3261^{*}$ \\
\hline FAT subcut. & -0.0272 & Cellular ferritin (H-FerritinActin) & 0.0099 \\
\hline Systolic BP & 0.2576 & Yield of monocytes & 0.1575 \\
\hline Diastolic BP & 0.1702 & MPO & -0.0109 \\
\hline IMT & 0.2032 & oxLDL & 0.0257 \\
\hline SI & 0,2524 & Anti oxLDL & -0.1055 \\
\hline$Y E M$ & 0.2303 & Hepcidin & $0.3365 *$ \\
\hline CAVI & -0.0670 & ICAM-1 & -0.0587 \\
\hline$A B I$ & $-0.3377^{*}$ & $M M P-1$ & 0.0883 \\
\hline$A I$ & 0.1674 & E-selectin & 0.1459 \\
\hline$P E P$ & 0.1393 & $v W F$ & -0.1787 \\
\hline ET & $-0.2748 *$ & VCAM & 0.1241 \\
\hline Total cholesterol & 0.0380 & CD163 & 0.0948 \\
\hline HDL cholesterol & -0.1018 & Glucose & 0.1494 \\
\hline LDL cholesterol & 0.0221 & Insulin & 0.0181 \\
\hline Triglycerides & 0.2034 & Homa IR & 0.0770 \\
\hline Apo A1 & 0.0264 & CRPus & 0.1913 \\
\hline Aро B & 0.0246 & WBCs & $0.2791 *$ \\
\hline $\operatorname{Lp}(a)$ & 0.0520 & $H G B$ & 0.1460 \\
\hline$P P$ & 0.2443 & HCT & 0.1142 \\
\hline $\mathrm{Fe}$ & 0.1371 & Baecke score - free time & $-0.2811^{*}$ \\
\hline TIBC & -0.1789 & Baecke score - work time & -0.0201 \\
\hline Ceruloplasmin & 0.0442 & Baecke score - household time & -0.0119 \\
\hline
\end{tabular}

* $\mathrm{P}<0.05$

Table 5. Multivariate regression analysis of LIP with correlated factors.

\begin{tabular}{lcc}
\hline & $\mathbf{b}$ & $\mathbf{P}$ \\
\hline Total & 1.4627 & 0.2584 \\
ABI & -0.1094 & 0.8312 \\
Baecke score - free time & -0.1240 & 0.2023 \\
VFT & 0.0809 & 0.0083 \\
ET & 0.0002 & 0.9505 \\
Hepcidin & 0.0080 & 0.5748 \\
WBCsfer. Rec./Ferritin & -0.1697 & 0.0875 \\
\hline
\end{tabular}


Table 6. Prediction of acute CVE* by the LIP values.

\begin{tabular}{lccc}
\hline & Upper tertile of LIP & Lower tertile of LIP & Total \\
\hline CVE + & 11 & 4 & 15 \\
CVE - & 6 & 13 & 19 \\
Total & 17 & 17 & 34 \\
& \multicolumn{2}{c}{ OR=5.96, P=0.0156 } \\
\hline
\end{tabular}

* Patients after AMI, stroke, or claudication.

\section{Discussion}

We found that patients with a history of CVE had significantly higher concentrations in their intracellular monocyte LIP than did healthy control individuals. The two groups did not statistically differ in the other estimated markers of iron metabolism; however, LIP showed a positive correlation with total body iron stores, expressed as the $\mathrm{TfR} / \mathrm{F}$ ratio. Because plasma ferritin and transferrin are acute-phase proteins, TfR/F may be a better marker of total body iron stores (Tuomainen et al. 1998) and has already used in our previous studies (Syrovatka et al. 2011, Syrovatka et al. 2009). LIP was also positively correlated with plasma hepcidin. This critical regulatory protein of iron metabolism is produced mostly by hepatocytes in response to high body iron content. Hepcidin directly binds to ferroportin, a major iron export protein in hepatocytes, enterocytes and macrophages, leading to decreased iron absorption (blocked export from enterocytes) as well as the inhibition of iron release from hepatocytes and macrophages. Hepcidin is another acute phase protein, often elevated by inflammation. To the best of our knowledge, none of the subjects in our study showed any signs or had a history of acute or chronic inflammatory disease within the 3 months prior to their examination. The number of WBCs was the only marker of inflammation that differed between the CVE patients and the controls. Moreover, WBCs significantly correlated with the LIP, suggesting that WBC numbers remain a sensitive indicator of activated immunity, even in patients with clinically stable cardiovascular diseases.

The LIP showed significant and independent correlations with markers of atherosclerotic progression (ABI) and arterial stiffness (ET). Thus, it seems likely that redox-active iron in monocytes triggers signaling cascades mediated by proinflammatory cytokines (TNF $\alpha$, IL-1 $\beta$, IL-6) that affect other cells participating on the formation of atherosclerotic plaques (endothelial cells, smooth muscle cells, thrombocytes, T-lymphocytes). Increased intracellular concentrations in the LIP in monocytes may thus contribute to the perpetuation of the atherosclerotic process. As expected, no differences were found in any of the estimated parameters of endothelial dysfunction (ICAM, VCAM, E-selectin, vWF) between the groups. These markers have short plasma half-lives (several min) and are usually not elevated in patients with chronic cardiovascular diseases. (Pearson et al. 2003).

We are aware of methodological limitations imposed by our choice of the procedure for the isolation of circulating monocytes. The separation of monocytes from other peripheral blood cells is generally difficult. For the LIP measurement we looked for a method that would be rapid and avoid the activation of monocytes, contamination with platelets, and any magnetic fields, as well as the lysis of erythrocytes, which would liberate a large quantity of iron. The flotation method used fulfilled these criteria; however, it gave us preparations of monocytes that on average were quite heavily contaminated with lymphocytes and to some extent with fragmented erythrocytes as well. It should be noted that even if the mean purity was merely $56 \%$, the majority of the signal during the spectrofluorimetric LIP measurement, perhaps approximately $80 \%$, still came from monocytes because these cells are larger than lymphocytes. Perhaps of even bigger concern, the yield and purity were also quite variable, which in our belief reflected genuine differences in the specific gravity of cells obtained from different subjects. The cell recovery is included in Table $1 \mathrm{D}$ as 'Yield of monocytes (\%)'. It is the number of isolated cells corrected for purity of each preparation as obtained from CD14 staining (\% monocytes), expressed as percentage of the original number of monocytes in the blood sample according to blood count. It combines information about purity of each preparation and cell recovery into a single parameter. 
Each subject was examined only once, so that we are no able to assess the variability of different runs in one donor. However, data from our pilot study focused on the isolation procedure demonstrated quite consistent results. Moreover, the LIP data displayed no statistical correlation with either the yield or the purity of the cell preparations, clearly indicating that our findings reported here are not due to experimental artifacts. A simple explanation might be that the differences in the LIP between the CVE patients and the controls might, in fact, reflect an abnormality in the cellular iron balance that is fairly general, occurring in various cell types, including lymphocytes and red blood cells. If this is the case, then the purity of the monocyte preparations is obviously not a significant issue. However, the same does not apply to the measurements of cellular ferritin by Western immunoblotting, where a statistically significant correlation with the yield and purity was observed, and any useful information in these data could be obscured by the heterogeneity of the cellular preparations.

The cohort of CVE patients had lower concentrations of plasma total cholesterol, LDL-cholesterol, and Apo B than were found in the healthy controls. This comes as no surprise because all CVE patients were on statin treatments. According to current guidelines (ESC/EAS Guidelines 2011, ACC/AHA Guideline 2014), therapy with statins is mandatory for all secondary prevention patients. This may also explain why no correlation of the LIP with plasma oxLDL was found. However, it should be added that the process of lipid oxidation occurs primarily in tissues, rather than in the plasma. Nevertheless, we found a correlation of iron stores with markers of both lipid as well as protein oxidation in our previous studies (Syrovatka et al. 2011, Syrovatka et al. 2009).

Patients with a history of CVE had significantly higher concentrations of plasma triglycerides and lower concentrations of HDL-cholesterol, indicating a higher incidence of metabolic syndrome than in the control group. Indeed, the CVE patients showed higher concentrations of plasma glucose, C-peptide, and insulin, as well as markers of insulin resistance (HOMA-IR). Although there was no difference in the waist circumference or BMI between the groups, significantly thicker VFT was found in the CVE patient cohort, as determined via precise ultrasound measurements of abdominal fat. Moreover, VFT was positively correlated with the LIP.
The correlation of elevated body iron stores with insulin resistance and obesity has been well known since 1992, when Salonen et al. published their first study on ferritin as a risk factor of acute myocardial infarction. This association has not yet been fully clarified. It is believed that increased iron consumption, namely heme iron in red meat, correlates with higher total energy intake levels in these individuals. On the other hand, heme iron may directly contribute to increased oxidative stress resulting in insulin resistance and obesity (Park et al. 2009). It has been demonstrated that oxidative stress in mammalian skeletal muscle leads to substantial insulin resistance due to impaired distal insulin signaling and glucose transport activity caused by a selective loss of the IRS-1 protein (Archuleta et al. 2009).

Until now it has not been fully elucidated which pathophysiological mechanism is mostly responsible for the LIP regulation in monocytes. Interventional studies using phlebotomy and/or reduced heme iron intake are needed to clarify whether elevated LIP levels could be effectively modified.

\section{Conclusion}

Patients with a history of CVE have significantly higher iron concentrations in the intracellular LIP of circulating monocytes than are found in healthy controls. No differences were found in other markers of iron metabolism. However, a regression analysis showed that the LIP positively correlated with TfR/F and plasma hepcidin, both markers of body iron stores. The LIP was also correlated with visceral fat tissue, which documents a possible role of iron in the development of insulin resistance. The significant and independent correlations of the LIP with markers of atherosclerotic progression (ABI) and arterial stiffness (ET) imply that the LIP could be a sensitive marker of atherosclerotic activity, even in patients with clinically stable cardiovascular diseases.

\section{Conflict of Interest}

There is no conflict of interest.

\section{Acknowledgements}

The study was supported by the Internal Grant Agency, Ministry of Health, Czech Republic, grant No.: NT13671-4/2012. The skillful technical assistance of Květa Hrubišová in the isolation of monocytes is acknowledged. 


\section{References}

ACC/AHA Guideline on the treatment of blood cholesterol to reduce atherosclerotic cardiovascular risk in adults. $J$ Am Coll Cardiol 63: 2889-2934, 2014.

ARCHULETA TL, LEMIEUX AM, SAENGSIRISUWAN V, TEACHEY MK, LINDBORG KA, KIM JS, HENRIKSEN EJ: Oxidant stress-induced loss of IRS-1 and IRS-2 proteins in rat skeletal muscle: role of p38 MAPK. Free Radic Biol Med 47: 1486-1493, 2009.

BAECKE JA, BUREMA J, FRIJTERS JE: A short questionnaire for the measurement of habitual physical activity in epidemiological studies. Am J Clin Nutr 36: 936-942, 1982.

BOVLUS CL: The role of iron in T cell development and autoimmunity. Autoimmun Rev 2: 73-78, 2003.

CAVALLINI MC, ROMAN MJ, BLANK SG, PINI R, PICKERING TG, DEVEREUX RB: Association of the auscultatory gap with vascular disease in hypertensive patients. Ann Intern Med 124: 877-883, 1996.

DE LUCIA ROLFE E, SLEIGH A, FINUCANE FM, BRAGE S, STOLK RP, COOPER C, SHARP SJ, WAREHAM NJ, ONG KK: Ultrasound measurements of visceral and subcutaneous abdominal thickness to predict abdominal adiposity among older men a women. Obesity 18: 625-631, 2010.

DEPALMA RG, HAYES VW, CAFFERATA HT, MOHAMMADPOUR HA, CHOW BK, ZACHARSKI LR, HALL MR: Cytokine signatures in atherosclerotic claudicants. J Surg Res 111: 215-221, 2003.

DOBIASOVA M, FROHLICH J: The plasma parameter log (TG/HDL-C) as an atherogenic index: correlation with lipoprotein particle size and esterification rate in apoB-lipoprotein-depleted plasma (FER(HDL)). Clin Biochem 34: 583-588, 2001.

EPSZTEJN S, KAKHLON O, GLICKSTEIN H, BREUER W, CABANTCHIK ZI: Fluorescence analysis of the labile iron pool of mammalian cells Anal Biochem 248: 31-40, 1997.

ESC/EAS Guidelines for the management of dyslipidaemias. Eur Heart J 32: 1769-1818, 2011.

FENG XB, YANG XQ, SHEN J: Influence of iron deficiency on serum IgG subclass and pneumococcal polysaccharides specific IgG subclass antibodies. Chin Med J 107: 813-816, 1994.

FLORINDO AA, LATORRE M DO RD DE O, SANTOS ECM DOS, NEGRÃO CE, AZEVEDO LF, SEGURADO AA: Validity and reliability of the Baecke questionnaire for the evaluation of habitual physical activity among people living with HIV/AIDS. Cad Saude Publica 22: 535-541, 2006.

GRAZIANI-BOWERING GM, GRAHAM J, FILION LG: A quick, easy and inexpensive method for the isolation of human peripheral blood monocytes J Immunol Meth 207: 157-168, 1997.

GREENBERG GR, WINTROBE MM: A labile iron pool. J Biol Chem 165: 397-398, 1946.

HABIB A, FINN AV: The role of iron metabolism as a mediator of macrophage inflammation and lipid handling in atherosclerosis. Front Pharmacol 5: 195, 2014.

Isolation of a monocyte-rich fraction from whole human blood by iodixanol barrier flotation. Axis-Shield Application Sheet C10, 5th edition, March 2013 (http://www.axis-shield-density-gradient-media.com).

JACOBS A: An intracellular transit iron pool. In: Ciba Foundation Symposium 51-Iron Metabolism. PORTER R, FITZSIMONS DW (eds), John Wiley \& Sons Ltd., 1977, pp 91-106.

KAKHLON O, CABANTCHIK Z: The labile iron pool: characterization, measurement, and participation in cellular processes. Free Radic Biol Med 33: 1037-1046, 2002.

KEMP JD: The role of iron and iron binding proteins in lymphocyte physiology and pathology. J Clin Immunol 13: 81-92, 1993.

HALLOU-LASCHET J, VARTHAMAN A, FORNASA G, COMPAIN C, GASTON AT, CLEMENT M, DUSSIOT M, LEVILLAIN O, GRAFF-DUBOIS S, NICOLETTI A, CALIGIURI G: Macrophage plasticity in experimental atherosclerosis. PLoS One 5: e8852, 2010.

KIECHL S, WILLEIT J, EGGER G, POEWE W, OBERHOLLENZER F: Body iron stores and the risk of carotid atherosclerosis: prospective results from the Bruneck study. Circulation 96: 3300-3307, 1997.

KONG W, DUAN X, SHI Z, CHANG Y: Iron metabolism in the mononuclear phagocyte system. Prog Nat Sci 18: 1197-1202, 2008.

KRUSZEWSKI M: Labile iron pool: the main determinant of cellular response to oxidative stress. Mutat Res 531: 81-92, 2003. 
LAUFFER RB: Iron depletion and coronary disease. Am Heart J 119: 1448-1449, 1990.

LEVY JC, MATTHEWS DR, HERMANS MP: Correct homeostasis model assessment (HOMA) evaluation uses the computer program. Diabetes Care 21: 2191-2192, 1998.

MENKE A, FERNANDEZ-REAL JM, MUNTNER P, GUALLAR E: The association of biomarkers of iron status with peripheral arterial disease in US adults. BMC Cardiovasc Disord 9: 34, 2009.

MITSUMURA H, SAKUTA K, BONO K, YAMAZAKI M, SENGOKU R, KONO Y, KAMIYAMA T, SUZUKI M, FURUHATA H, IGUCHI Y: Stiffness parameter $\beta$ of cardioembolism measured by carotid ultrasound was lower than other stroke subtypes. J Stroke Cerebrovasc Dis 23: 1391-1395, 2014.

PANG JH, JIANG MJ, CHEN YL, WANG FW, WANG DL, CHU SH: Increased ferritin gene expression in atherosclerotic lesions. J Clin Invest 97: 2204-2212, 1996.

PARK K, GROSS M, LEE DH, HOLVOET P, HIMES JH, SHIKANY JM: Oxidative stress and insulin resistance: the coronary artery risk development in young adults study. Diabetes Care 32: 1302-1307, 2009.

PEARSON TA, MENSAH GA, ALEXANDER RW, ANDERSON JL, CANNON RO 3RD, CRIQUI M, FADL YY, FORTMANN SP, HONG Y, MYERS GL, ET AL.: Markers of inflammation and cardiovascular disease. Circulation 107: 499-511, 2003.

SALONEN JT, NYYSSONEN K, KORPELA H, TUOMILEHTO J, SEPPANEN R, SALONEN R: High stored iron levels are associated with excess risk of myocardial infarction in eastern Finnish men. Circulation 86: 803-811, 1992.

SMITH JD, TROGAN E, GINSBERG M, GRIGAUX C, TIAN J, MIYATA M: Decreased atherosclerosis in mice deficient in both macrophage colony-stimulating factor (op) and apolipoprotein E. Proc Natl Acad Sci U S A 92: 8264-8268, 1995.

STEIN JH, KORCARZ CE, HURST RT, LONN E, KENDALL CB, MOHLER ER: Use of carotid ultrasound to identify subclinical vascular disease and evaluate cardiovascular disease risk: a consensus statement from the American Society of Echocardiography Carotid Intima-Media Thickness Task Force. Endorsed by the Society for Vascular Medicine. J Am Soc Echocardiogr 21: 93-111. quiz 189-190, 2008.

SULLIVAN JL: Iron and the sex difference in heart disease risk. Lancet 1: 1293-1294, 1981.

SYROVATKA P, KRAML P, HULIKOVA K, FIALOVA L, VEJRAZKA M, CRKOVSKA J, POTOCKOVA J, ANDEL M: Iron stores are associated with asymptomatic atherosclerosis in healthy men of primary prevention. Eur J Clin Invest 41: 846-853, 2011.

SYROVATKA P, KRAML P, POTOCKOVA J, FIALOVA L, VEJRAZKA M, CRKOVSKA J, ANDEL M: Relationship between increased body iron stores, oxidative stress and insulin resistance in healthy men. Ann Nutr Metab 54: 268-274, 2009.

TUOMAINEN TP, PUNNONEN K, NYYSSÖNEN K, SALONEN JT: Association between body iron stores and the risk of acute myocardial infarction in men. Circulation 97: 1461-1466, 1998.

YUAN X, LI W, BAIRD SK, CARLSSON M, MELEFORS O: Secretion of ferritin by iron-laden macrophages and influence of lipoproteins. Free Radic Res 38: 1133-1142, 2004. 